Pharmacother. 5:1385-1398.

14. Tornvall, P., and Walldius, G. 1991. A comparison between nicotinic acid and acipimox in hypertriglyceridaemia - effects on serum lipids, lipoproteins, glucose tolerance and tolerability. J. Intern. Med. 230:415-421.

15. Schaub, A., Futterer, A., and Pfeffer, K. 2001. PUMA-G, an IFN-gamma-inducible gene in macrophages is a novel member of the seven transmembrane spanning receptor superfamily. Eur. J. Immunol. 31:3714-3725.

16. Carlson, L.A. 1978. Nicotinic acid and inhibition of fat mobilizing lipolysis. Present status of effects on lipid metabolism. Adv. Exp. Med. Biol. 109:225-238.

17. Taggart, A.K.P., et al. 2005. (D)- $\beta$-Hydroxybutyrate inhibits adipocyte lipolysis via the nicotinic acid receptor PUMA-G. J. Biol. Chem. 280:26649-26652.

18. Jin, F.Y., Kamanna, V.S., and Kashyap, M.L. 1999. Niacin accelerates intracellular ApoB degradation by inhibiting triacylglycerol synthesis in human hepatoblastoma (HepG2) cells. Arterioscler. Thromb. Vasc. Biol. 19:1051-1059.

19. Jin, F.Y., Kamanna, V.S., and Kashyap, M.L. 1997. Niacin decreases the removal of high-density lipo- protein apolipoprotein A-I but not cholesterol ester by HepG2 cells. Implications for reverse cholesterol transport. Arterioscler. Thromb. Vasc. Biol. 17:2020-2028.

20. Ganji, S.H., Kamanna, V.S., and Kashyap, M.L. 2003. Niacin and cholesterol: role in cardiovascular disease. J. Nutr. Biochem. 14:298-305.

21. Rubric, T., Trottmann, M., and Lorenz, R.L. 2004. Stimulation of CD36 and the key effector of reverse cholesterol transport ATP-binding cassette A1 in monocytoid cells by niacin. Biochem. Pharmacol. 67:411-419.

\title{
Desperately seeking sugar: glial cells as hypoglycemia sensors
}

\author{
Amira Klip ${ }^{1}$ and Meredith Hawkins ${ }^{2}$ \\ ${ }^{1}$ Cell Biology Programme, The Hospital for Sick Children, Toronto, Ontario, Canada. 2Division of Endocrinology, Department of Medicine,
} Albert Einstein College of Medicine of Yeshiva University, New York, New York, USA.

\begin{abstract}
A life-saving response to hypoglycemia requires rapid sensing of decreases in glycemia and consequent brisk glucagon secretion. Preceding studies have shown that mice lacking glucose transporter type 2 (GLUT2) lose this response. In this issue of the JCI, Marty et al. report that glucose sensing and consequent pancreatic glucagon secretion are restored by re-expression of GLUT2 in glial but not neuronal cells (see the related article beginning on page 3545). A new, glucose-sensing role is ascribed to GLUT2expressing glial cells.
\end{abstract}

\section{Central responses to hypoglycemia}

Hypoglycemia is a life-threatening condition in which blood glucose can drop to levels that jeopardize brain function. Insulin and antihyperglycemic therapies are major causes of dangerous hypoglycemic episodes in diabetic individuals. With the global prevalence of the disease and ambitious goals for blood sugar reduction, hypoglycemia has become a clinically significant health issue. The ability to rapidly sense a drop in circulating glucose and induce a counterregulatory response forms the cornerstone of the body's life-saving responses to hypoglycemia. Accordingly, several systems participate in glucose sensing and the evoked secretion of glucagon and adrenalin. How is hypoglycemia detected in the first place? The following have been proposed: (a) glucose levels may be sensed by the glucagon-secreting, pancreatic $\alpha$ cells; (b) low intrapancreatic insulin levels may regulate glucagon secretion; and (c) the

Nonstandard abbreviations used: GLUT2, glucose transporter type 2; NTS, nucleus of the tractus solitarius.

Conflict of interest: The authors have declared that no conflict of interest exists.

Citation for this article: J. Clin. Invest. 115:3403-3405 (2005). doi:10.1172/JCI27208.
CNS may sense the drop in glucose and elicit secretory hormonal responses. Hypoglycemia-evoked glucagon and epinephrine secretion are similarly rapid (within minutes) and activated by comparable blood glucose nadirs (3.6-3.9 mM) (1). However, these hormonal responses may be activated by different mechanisms, each involving the CNS. Glucagon secretion elicited by hypoglycemia is normal in a neuronal insulin-receptor knockout (NIRKO) mouse model, yet the sympathoadrenal response is markedly impaired (2). In contrast, mice lacking ATP-sensitive $\mathrm{K}^{+}$channels display a severe defect in glucagon secretion (despite normal $\alpha$ cell function) yet secrete epinephrine normally in response to hypoglycemia (3). In people with type 1 diabetes, the loss of glucagon secretion with hypoglycemia occurs early in the disease course while the defect in epinephrine secretion becomes manifest later (1).

Injecting glucoprivic agents such as 2-deoxyglucose into either the ventromedial hypothalamus or certain brainstem sites activates neurohumoral counterregulatory responses similar to those seen during systemic hypoglycemia $(4,5)$. Subsequent efferent output to the $\alpha$ cells involves the autonomic nervous system (6). The presence of both glucose transporter type 2 (GLUT2) and glucokinase in some neurons and astrocytes in glucose-sensitive brain regions led to the concept of a central glucose sensor similar to that in pancreatic $\beta$ cells (7). However, although GLUT2 is expressed in up to one-third of glucose-sensing neurons, an equal number of non-glucose-sensing neurons also express this transporter (8). In this issue of the JCI, Marty et al. show that mice lacking GLUT2 are unable to respond to hypoglycemia with increased glucagon secretion despite restoration of glucose-stimulated insulin secretion by $\beta$ cell-selective Glut 1 overexpression (RipGlut1;Glut2-/- mice) (9). Therefore, a picture emerges in which, in addition to hypoglycemia detection by glucose flux into $\alpha$ cells and the intrapancreatic insulin relay, central detection of glucose is paramount and generates an efferent glucagon-releasing response involving glucose transport into glial cells.

\section{Astrocytes/glial cells: first responders to hypoglycemic emergencies?}

At the core of this study (9) is the observation that transgenic overexpression of GLUT2 in glial cells but not neurons restored this vital response in RipGlut1;Glut2-/- mice. Such central detection requires glial cells and likely involves glial cell-neuron communication in central locations, including the nucleus of the tractus solitarius (NTS) and the dorsal motor nucleus of the vagus (Figure 1). 




Implicating glial cells as hypoglycemic sensors is consistent with the observation that glial cell toxins prevent activation of neurons by glucoprivic agents in both the NTS and the hypothalamus (10). Other recent observations support the role of glial cell glucose metabolism, likely via vagal autonomic outflow, in controlling important systemic processes. Metabolism of glucose to lactate in astrocytes is required to activate neuronal ATP-sensitive $\mathrm{K}^{+}$channels (via pyruvate formation) and ultimately mediate the suppressive effects of glucose on hepatic glucose production (11). This is in line with the concept of an astrocyte-neuron lactate shuttle (12), in which metabolism of glucose by astrocytes generates extracellular lactate that serves as substrate for neuronal pyruvate synthesis. However, since systemic responses to hyperglycemia and hypoglycemia differ substantially $(1,13)$, central responses to increased glucose versus hypoglycemia may involve distinct mechanisms.

Another interesting observation is the lack of participation of neurons in direct glucose sensing (9). Marty et al. exclude neurons as the primary sensors of brain glucose levels due to the lack of restoration of hypoglycemia-induced glucagon release in RipGlut1;Glut2-/- mice upon transgenic expression of the Glut2 gene via the synapsin promoter (9). Given the diversity of neuronal gene expression patterns, is it possible that a participating neuronal subpopulation does not activate this promoter?

\section{GLUT2: a sensor of hyperglycemia and hypoglycemia?}

GLUT2 is biologically ideal to act as the pancreatic $\beta$ cell glucose sensor during hyperglycemia, allowing for a sudden increase in circulating glucose to translate into a rise in intracellular glucose metabolite and ATP levels. Indeed, when the level of glycemia rises during a meal, GLUT2 remains “open for business” while other glucose transporters are saturated. This is because the $\mathrm{K}_{\mathrm{m}}$ for glucose of GLUT2 is $17 \mathrm{mM}$, compared with $3 \mathrm{mM}$ for the abundantly expressed GLUT1 (14). In fact, GLUT2 has the lowest affinity for glucose of all the members of class I glucose transporters (including the neuronally expressed GLUT3, which has a $\mathrm{K}_{\mathrm{m}}$ of approximately $1.4 \mathrm{mM}$, and the muscle- and fat-expressed GLUT4, which has a $\mathrm{K}_{\mathrm{m}}$ of $5 \mathrm{mM}$ and is also expressed in several brain regions) (14). Of note, different brain regions are exposed to distinct

\section{Figure 1}

Proposed glial-neuronal loop at work in central sensing of hypoglycemia via GLUT2, based on the study by Marty et al. in this issue (9). This scheme illustrates the pivotal role of GLUT2 in glial cells in first-hand detection of hypoglycemia. How these specific glial cells then connect to neurons within the brainstem (likely in the NTS and the dorsal motor nucleus of the vagus) to relay information is unknown but may involve the lactate shuttle as well as signaling via the Kir6.2 ATP-regulated $\mathrm{K}^{+}$ channel (not illustrated). The drop in glycemia may also be directly sensed by neurons and pancreatic $\alpha$ and $\beta$ cells but not through GLUT2 (the transporter/detectors involved are so far unknown). Ultimately, autonomic nervous signals and the drop in intraislet insulin levels promote glucagon secretion.

glucose levels within a large span ranging from $0.5-10 \mathrm{mM}(8)$.

One of the most surprising findings by Marty et al. (9) is that glucose flux through GLUT2 may be sufficiently robust in the hypoglycemia-sensing glial cells to produce significant changes in intracellular metabolites at low-glucose concentrations. Glial cells are rich in GLUT1, and presumably a substantial portion of glucose influx occurs through this transporter (15). For GLUT2 to elicit a signal within hypoglycemia-sensing glial cells, GLUT2 rather than GLUT1 would have to dictate transport. It is interesting to note that insulin levels are markedly reduced in RipGlut1;Glut2-/mice in both fed and fasted states (16). Could these chronically low insulin levels downregulate Glut1 expression in glial cells (and elsewhere), thereby contributing to defective glucose sensing and emphasizing the role of GLUT2? Alternatively, could GLUT2 be a determinant for differentiation or survival during embryogenesis of a particular subpopulation of glial cells that transport glucose through another GLUT type (Figure 1)? Cell-sorting strategies based on these alternative predictions would be potentially useful in isolating the proposed hypoglycemiasensing glial cell population. 


\section{Next step: a molecular mechanism of glucose sensing?}

How does GLUT2 act as a hypoglycemia sensor? Is it possible that GLUT2 functions as a structural detector in conjunction with glucokinase and/or the ATP-sensitive $\mathrm{K}^{+}$channel Kir6.2? If ATP or glucose metabolite levels are the intracellular readouts of extracellular glucose levels, then lack of GLUT2 should mimic hypoglycemia, and Glut2 $2^{-/}$mice should secrete elevated glucagon levels. This is indeed seen under basal conditions in the RipGlut1;Glut2-/- mice; however, they lack the glucagon response elicited by hypoglycemia or the glucoprivic signal of 2-deoxyglucose. Indeed, the high basal glucagon levels and lack of secretory response to hypoglycemia in RipGlut1;Glut2-/- mice might raise the hypothesis that hypoglycemia signaling is the default "on" pathway, which is suppressed by the sensing of adequate levels of glucose. These two consequences of Glut2 deletion (high-tonic glucagonemia and loss of response to hypoglycemia) coexist in RipGlut1;Glut2-/mice of mixed genetic lineage, but only the former is maintained when mice are backcrossed into the C57BL/ 6 background. The different genetic backgrounds may help identify other genes that sustain the response to hypoglycemia.

Is it possible that transgenic (and hence supraphysiologic) overexpression of Glut2 in glial cells may have corrected the defect in glucose transport, even if GLUT2 were not normally the dominant transporter in these cells? As the authors point out (9), a significant role of GLUT1 in glucose sensing could explain the normal glucagon response to hypoglycemia seen in the C57BL/ 6 background.

Since GLUT2 does not require insulin for activation, glucose sensing by GLUT2 alone would not explain the apparent lack of glucose sensing in diabetic ketoacidosis (DKA), a state of complete insulin deficiency. Individuals in DKA often crave food and sweet beverages and have elevated glucagon levels despite severely high blood glucose concentrations (17). One might speculate that in such hypoinsulinemic patients, the brain senses hypoglycemia in the presence of high circulating glucose levels. It would be interesting to examine the role of glial cells and of GLUT2 in particular in the absence of insulin. Is it possible that at least basal insulin levels are required for normal central glucose sensing? If so, other glucose transport mechanisms might also be required for normal glucose sensing. GLUT1 and GLUT3 are the dominant glucose transporters in astrocytes and neurons, respectively. Both transporters have been studied extensively in myocytes, in which they are mobilized to the plasma membrane in response to insulin (18).

\section{Conclusions and queries}

Perhaps the most far-reaching conclusion of this valuable study (9) is the determination that glial cells are key elements in the direct sensing of glucose. Therefore, this implicates a glial-neuronal loop in the ensuing efferent response that activates glucagon secretion. This study also proposes the participation of GLUT2 as a hypoglycemic sensor in addition to its more familiar role as a hyperglycemia detector. Exciting new challenges lie ahead, including the identification and localization of the precise GLUT2-endowed glial cells involved, the molecular details of the mechanism of hypoglycemia sensing, and ultimately the identification of the connecting neurons that complete the circuit to the pancreatic $\alpha$ cell.

\section{Acknowledgments}

The authors thank Tony Lam, Luciano Rossetti, and Harry Shamoon for helpful discussions.

Address correspondence to: Amira Klip, Cell Biology Programme, The Hospital for Sick Children, 555 University Avenue, Toronto M5G 1X8, Ontario, Canada. Phone: (416) 813-6392; Fax: (416) 8135028; E-mail: amira@sickkids.ca.
1. Cryer, P.E., Davis, S.N., and Shamoon, H. 2003. Hypoglycemia in diabetes. Diabetes Care. 26:1902-1912.

2. Fisher, S.J., Bruning, J.C., Lannon, S., and Kahn, C.R. 2005. Insulin signaling in the central nervous system is critical for the normal sympathoadrenal response to hypoglycemia. Diabetes. 54:1447-1451.

3. Miki, T., et al. 2001. ATP-sensitive $\mathrm{K}+$ channels in the hypothalamus are essential for the maintenance of glucose homeostasis. Nat. Neurosci. 4:507-512.

4. Borg, W.P., Sherwin, R.S., During, M.J., Borg, M.A., and Shulman, G.I. 1995. Local ventromedial hypothalamic glucopenia triggers counterregulatory hormone release. Diabetes. 44:180-184.

5. Ritter, S., Dinh, T.T., and Zhang, Y. 2000. Localization of hindbrain glucoreceptive sites controlling food intake and blood glucose. Brain Res. 856:37-47.

6. Havel, P.J., Veith, R.C., Dunning, B.E., and Taborsky, G.J., Jr. 1991. Role for autonomic nervous system to increase pancreatic glucagon secretion during marked insulin-induced hypoglycemia in dogs. Diabetes. 40:1107-1114.

7. Penicaud, L., Leloup, C., Lorsignol, A., Alquier, T., and Guillod, E. 2002. Brain glucose sensing mechanism and glucose homeostasis. Curr. Opin. Clin. Nutr. Metab. Care. 5:539-543.

8. Levin, B.E., Routh, V.H., Kang, L., Sanders, N.M., and Dunn-Meynell, A.A. 2004. Neuronal glucosensing: what do we know after 50 years? Diabetes. 53:2521-2528.

9. Marty, N., et al. 2005. Regulation of glucagon secretion by glucose transporter type 2 (glut2) and astrocyte-dependent glucose sensors. J. Clin. Invest. 115:3545-3553. doi:10.1172/JCI26309.

10. Young, J.K., Baker, J.H., and Montes, M.I. 2000. The brain response to 2-deoxy glucose is blocked by a glial drug. Pharmacol. Biochem. Behav. 67:233-239.

11. Lam, T.K., Gutierrez-Juarez, R., Pocai, A., and Rossetti, L. 2005. Regulation of blood glucose by hypothalamic pyruvate metabolism. Science. 309:943-947.

12. Pellerin, L., et al. 1998. Evidence supporting the existence of an activity-dependent astrocyte-neuron lactate shuttle. Dev. Neurosci. 20:291-299.

13. Tonelli, J., Kishore, P., Lee, D.E., and Hawkins, M. 2005. The regulation of glucose effectiveness: how glucose modulates its own production. Curr. Opin. Clin. Nutr. Metab. Care. 8:450-456.

14. Uldry, M., and Thorens, B. 2004. The SLC2 family of facilitated hexose and polyol transporters. Pflugers. Arch. 447:480-489.

15. Maher, F., Vannucci, S.J., and Simpson, I.A. 1993. Glucose transporter isoforms in brain: absence of GLUT3 from the blood-brain barrier. J. Cereb. Blood Flow Metab. 13:342-345.

16. Burcelin, R., and Thorens, B. 2001. Evidence that extrapancreatic GLUT2-dependent glucose sensors control glucagon secretion. Diabetes. 50:1282-1289.

17. Cugini, P., et al. 1996. Hunger sensation in patients with compensated and uncompensated type 1 and type 2 diabetes mellitus. Int. J. Eat. Disord. 20:85-98.

18. Wilson, C.M., Mitsumoto, Y., Maher, F., and Klip, A. 1995. Regulation of cell surface GLUT1, GLUT3, and GLUT4 by insulin and IGF-I in L6 myotubes. FEBS Lett. 368:19-22. 